\title{
Fine mapping of foxglove aphid (Aulacorthum solani) resistance gene Raso1 in soybean and its effect on tolerance to Soybean dwarf virus transmitted by foxglove aphid
}

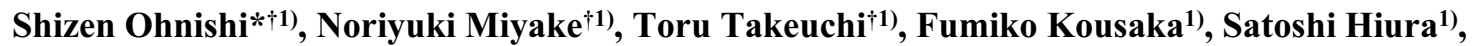 \\ Osamu Kanehira1), Miki Saito'), Takashi Sayama ${ }^{2,3)}$, Ayako Higashi' ${ }^{3)}$, Masao Ishimoto ${ }^{2,3)}$, \\ Yoshinori Tanaka') and Shohei Fujita ${ }^{1)}$ \\ 1) Hokkaido Research Organization Central Agricultural Experiment Station, Naganuma, Yubari, Hokkaido 069-1395, Japan \\ 2) National Institute of Agrobiological Sciences, 2-1-2 Kannondai, Tsukuba, Ibaraki 305-8602, Japan \\ 3) National Agricultural Research Center for Hokkaido Region, Hitsujigaoka, Toyohira, Sapporo, Hokkaido 062-8555, Japan
}

\begin{abstract}
Soybean dwarf virus (SbDV) causes serious dwarfing, yellowing and sterility in soybean (Glycine max). The soybean cv. Adams is tolerant to SbDV infection in the field and exhibits antibiosis to foxglove aphid (Aulacorthum solani), which transmits SbDV. This antibiosis (termed "aphid resistance") is required for tolerance to SbDV in the field in segregated progenies of Adams. A major quantitative trait locus, Rasol, is reported for foxglove aphid resistance. Our objectives were to fine map Rasol and to reveal whether Rasol alone is sufficient to confer both aphid resistance and SbDV tolerance. We introduced Rasol into cv. Toyomusume by backcrossing and investigated the degree of aphid antibiosis to foxglove aphid and the degree of tolerance to SbDV in the field. All Rasol-introduced backcross lines showed aphid resistance. Interestingly, only one Rasol-introduced backcross line (TM-1386) showed tolerance to SbDV in the field. The results demonstrated Rasol alone is sufficient to confer aphid resistance but insufficient for SbDV tolerance. Tolerance to SbDV was indicated to require additional gene(s) to Raso1. Additionally, Raso1 was mapped to a $63-\mathrm{kb}$ interval on chromosome 3 of the Williams 82 sequence assembly (Glyma1). This interval includes a nucleotide-binding site-leucine-rich repeat encoding gene and two other genes in the Williams 82 soybean genome sequence.
\end{abstract}

Key Words: soybean, foxglove aphid, aphid resistance, soybean dwarf virus, NBS-LRR.

\section{Introduction}

Soybean dwarf virus (SbDV), a member of the Luteoviridae, is known to infect to soybean (Glycine max), red and white clovers (Trifolium pratense and T. repens) and other legume species (Tamada 1970). Infection of soybean by SbDV is widespread in northern Japan (Tamada 1975) and the United States (Thekkeveetil et al. 2007). In nature, SbDV is persistently transmitted by several aphid species (Gildow et al. 2000). In northern Japan, the foxglove aphid (Aulacorthum solani) transmits SbDV (Mikoshiba et al. 1995) from SbDV-infected red and white clovers to soybean during spring to summer. Infection by SbDV causes serious dwarfing, yellowing and sterility, and leads to significant yield losses in soybean production. To avoid damage induced by SbDV, soybean breeders have attempted to introduced SbDV resistant/tolerant germplasm. An old American culti-

Communicated by Dunean Vanghan

Received June 28, 2011. Accepted September 26, 2011.

*Corresponding author (e-mail: ohnishi-shizen@hro.or.jp)

$\dagger$ These authors contributed equally to this work var, 'Adams' (PI 548502), was found to show lower SbDV symptom incidence in the field. Adams suffers SbDV infection to a limited extent. However, the virus infection rate and symptom incidence are lower than other cultivars (Tanimura and Tamada 1976). In this paper, we termed this partial resistance to SbDV in the field "tolerance". Interestingly, the number of foxglove aphids observed on Adams is lower than that on other cultivars in the field (Kanehira 1978). Moreover, young Adams plants exhibited antibiosis to the foxglove aphid in a no-choice test (Jinno et al. 1997). We termed this aphid antibiosis "aphid resistance". Resistance to foxglove aphid was required for SbDV tolerance in the field when SbDV tolerance and aphid resistance were investigated in segregated progenies of Adams (Araki et al. 1997).

Aphid resistance is known among many plant species, including tomato (Solanum lycopersicum) (Rossi et al. 1998), wheat (Triticum aestivum) (Liu et al. 2005), barley (Hordeum vulgare) (Mittal et al. 2008), melon (Cucumis melo) (Brotman et al. 2002), barrel clover (Medicago truncatula) (Klingler et al. 2005), maize (Zea mays) (So et al. 2010) and soybean (Kim et al. 2010a, 2010b, Zhang et al. 
2010). Some aphid resistance genes have been cloned or fine mapped. In most cases, the resistance genes or the candidate of the resistance gene are a typical R-gene containing a leucine-rich repeat (Dogimont et al. 2010, Kim et al. 2010a, 2010b, Klingler et al. 2005, Milligan et al. 1998, Rossi et al. 1998, Smith and Boyko 2007). Thus aphid resistance in most plants seems to be controlled by the same mechanism as for other pest and disease resistance.

A previous report showed that aphid resistance in Adams is controlled by a quantitative trait locus (QTL) named Rasol (Kamiya et al. 2008), which has a relatively high contribution rate $\left(\mathrm{r}^{2}=0.32\right)$. Using 204 RILs, Rasol was detected on the $3.7 \mathrm{cM}$ region between Satt009 and Satt530 on chromosome 3 with a lod score of 16.0. The QTL analysis showed Rasol was essential for aphid resistance, but it is not clear whether Rasol is solely responsible for the aphid resistance. Similarly, based on previous reports, Rasol was deduced to be required for the SbDV tolerance phenotype of Adams, but it is not clear whether Rasol alone is sufficient to confer SbDV tolerance. In this study, we fine-mapped Rasol and investigated whether Rasol alone is sufficient to confer both aphid resistance and SbDV tolerance phenotype in Adams.

\section{Materials and Methods}

\section{Plant material}

Soybean cv. Adams (PI 548502) shows both foxglove aphid resistance and SbDV tolerance in the field (Jinno et al. 1997, Kanehira et al. 1978, Tanimura and Tamada 1976). Shokukei-10 is a breeding line which shows both foxglove aphid resistance and SbDV tolerance in the field (Fig. 1). Shokukei-10 was derived by crossing Adams with Mechita, a Russian cultivar (Fig. 1A). We introduced the aphid resistance QTL Rasol by backcrossing to Toyomusume, which is a commercial cultivar in northern Japan. Four backcrosses were performed using Toyomusume as the recurrent parent and Shokukei-10 as the donor of Raso1. A total of 9 backcross lines were developed from $9 \mathrm{BC}_{4} \mathrm{~F}_{1}$ plants. The Toyomusume backcrossed lines (TM-lines) were named TM-1382, TM-1386, TM-1387, TM-1388, TM-1394, TM1397, TM-1402, TM-1404 and TM-1409. The simple sequence repeats (SSR) markers Satt152, Satt009 and Satt530 were used to introduce Rasol based on a previous report (Kamiya et al. 2008). Primer sequences and positions of SSR markers on the genome sequences are described in SoyBase (www.soybase.org). We started the backcrossing by crossing Toyomusume to $F_{1}$ plants generated from a cross between Shokukei-10 and Toyomusume. Then, Toyomusume was crossed to a population consisting of a mixture of 4 independent $\mathrm{BC}_{1} \mathrm{~F}_{1}$ plants. To generate $\mathrm{BC}_{3} \mathrm{~F}_{1}$ and $\mathrm{BC}_{4} \mathrm{~F}_{1}$ plants, 12 randomly selected individual $\mathrm{BC}_{2} \mathrm{~F}_{1}$ and $\mathrm{BC}_{4} \mathrm{~F}_{1}$ plants were used. Thus, the final $9 \mathrm{BC}_{4}$ lines were partially independent. We used mapping populations C1905 and C1950, which were originally made for a breeding program and therefore have complex pedigrees. The
A

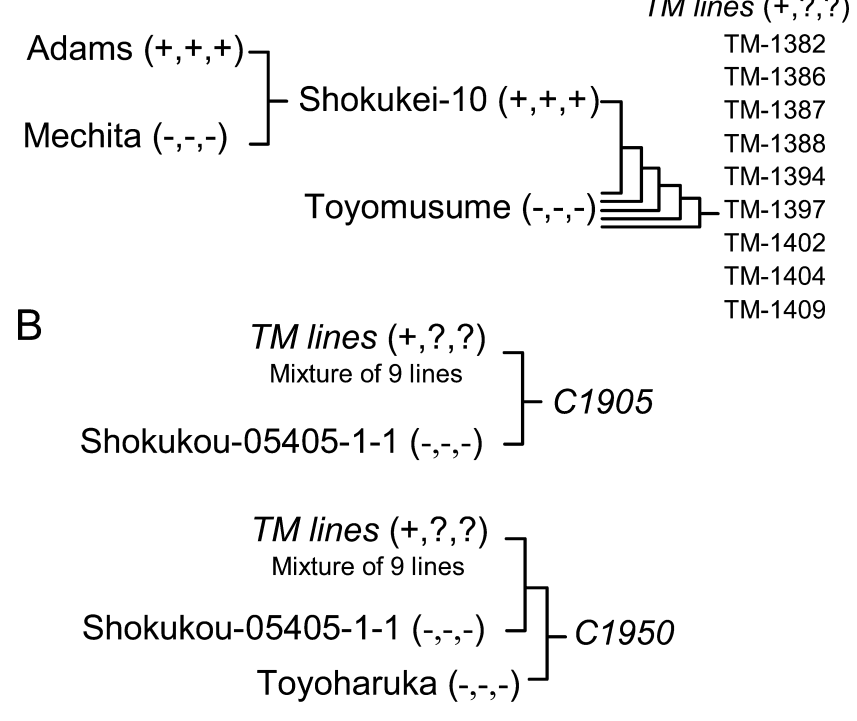

Fig. 1. Schematic representation of development of experimental lines. The plus (+) or minus (-) signs in parentheses represent the presence or absence of QTL Raso 1, aphid resistance, and SbDV tolerance, respectively, derived from Adams. Question marks represent "determined in this paper". (A) TM-lines are Toyomusume-backcrossed lines with the putative aphid resistance QTL Raso1. (B) C1905 and C1950 are mapping populations. C1905 and C1950 were originally made for a breeding program, but we used them for fine mapping. C1950 was derived from a 3-way crossing of $F_{1}$ plants of C1905 and Toyoharuka.

population C1905 was derived by crossing the population derived from mixing the 9 TM-lines described above in the $\mathrm{F}_{4}$ generation to an experimental line, Shokukou-05405-1-1 (Fig. 1B). Shokukou-05405-1-1 is a breeding line derived from backcrossing Tyokomachi, a cultivar in northern Japan, to an Indonesian cultivar, Wilis. The mapping population C1950 was generated by crossing $\mathrm{F}_{1}$ plants of C1905 and Toyoharuka, a cultivar in northern Japan. Crossing Toyoharuka was for breeding purposes and not essential for mapping. Shokukou-05405-1-1 and Toyoharuka do not exhibit aphid resistance.

\section{Evaluation of aphid resistance}

For nine TM-lines (TM-1382, TM-1386, TM-1387, TM1388, TM-1394, TM-1397, TM-1402, TM-1404 and TM1409), a no-choice test was performed according to a previous report (Jinno et al. 1997) to determine the degree of aphid antibiosis. Foxglove aphids (Acyrthosiphon solani) were collected at Naganuma, Japan $\left(43.017^{\circ} \mathrm{N}, 141.695^{\circ} \mathrm{E}\right)$, and were maintained on fresh leaves of the aphid-susceptible soybean cultivar Chusei-hikarikuro. Three soybean seeds were sown in unglazed pots (height $12 \mathrm{~cm}$; diameter $10 \mathrm{~cm}$ ) filled with potting soil. Seedlings were thinned to one per pot to synchronize the plant growth stage. A single sevenday-old aphid was settled per plant when the first true leaf was 3-5 mm long (cotyledons were not completely opened). After settlement, plants were grown in a plant incubator 
Table 1. Primer sequences and location of markers on chromosome 3 of the Williams 82 soybean genome sequence assembly (Glyma1)

\begin{tabular}{llll}
\hline \hline Marker Name & Location $^{a}$ & Forward primer & Reverse primer \\
\hline Gm03-01 & $4,811,664$ & AACCGTCGCTTAATCGTGATA & CCCTGAAAGCATAAAAAGTGG \\
Gm03-03 & $5,448,257$ & CATAAACTTTGGTCATGCTTAACTG & ACCTTGACCAAACATTCTTGA \\
Gm03-06 & $4,683,586$ & CTTGTATATATTACATTAAAATTGGAG & TGATGAAGAGTGACTACAGTTGAG \\
Gm03-07 & $4,697,806$ & AAAAACCTTGATTTGTTCTTCA & CAAAGGTTGTTACAGAAATTCAA \\
Gm03-09 & $4,613,933$ & CCATTCTAAGATGATTATTTACCTG & GACGCATGAAGACGATTTT \\
Gm03-10 & $4,637,354$ & CGGACAACCAAATCTCAAT & GAAAGCATGGAATAAACGAA \\
Gm03-11 & $4,661,084$ & GTGGCCACATGAAAATACAG & TCCTGGTGGACTTGCTAATA \\
Gm03-12 & $4,724,159$ & AAAAAGGCTAAAAGTATATTTCTGT & TGATACCATGATGTTTATATTTTT \\
\hline
\end{tabular}

${ }^{a}$ Nucleotide numbers from the end of chromosome to the forward primer.

maintained at $15^{\circ} \mathrm{C}$ with a $16 \mathrm{~h} / 8 \mathrm{~h}$ (light/dark) photoperiod. Each plant was covered with a mesh cage. The number of aphids on each plant was counted 14 days after settlement. The same procedure was adopted for fine mapping of Rasol.

\section{Evaluation of SbDV tolerance in the field}

To evaluate SbDV tolerance, we selected 3 TM-lines (TM-1394, TM-1382 and TM-1386) that differ in degree of aphid antibiosis. Field evaluations of SbDV tolerance were performed in Date City, Japan $\left(42.505^{\circ} \mathrm{N}, 140.889^{\circ} \mathrm{E}\right)$, a region with a severe SbDV endemic infection. There are two virus strains SbDV-D and SbDV-Y, which cause yellowing and dwarfing symptoms in soybean (Tamada 1973). Both strains are observed in Date. In this region, no viral disease except $\mathrm{SbDV}$ was observed in soybean, and thus the dwarfing or yellowing symptoms were likely caused by SbDV. Soybean lines were sown on 13 May 2008 and 8 May 2009. Soybean fields were managed according to the local custom except that pesticide applications for control of aphids were omitted. Endemic SbDV symptoms on soybean were first observed about 50 days after sowing. Soybean plants with dwarfing or yellowing symptoms were counted and the incidence rate against the total number of the tested plants was calculated as "virus symptom incidence". Four replications of each line were grown and each $1.32 \mathrm{~m}^{2}$ plot contained 22 plants. Virus symptom severity was scored according to a previous report (Mikoshiba et al. 1990) on 3 November 2009, 113 days after sowing.

\section{Mapping and marker assay}

From the mapping populations described above, a total of $669 \mathrm{~F}_{2}$ plants for $\mathrm{C} 1905$ and $576 \mathrm{~F}_{2}$ plants for $\mathrm{C} 1950$ were screened with the SSR markers Satt009 and Satt530, which flank the QTL Rasol (Kamiya et al. 2008). We selected 59 plants for $\mathrm{C} 1905$ and 34 plants for $\mathrm{C} 1950$ in which a recombination event between the markers was confirmed. The harvested plants were used to produce the next generation in a greenhouse and one homozygous plant per recombination event was selected and grown to produce seed. For further genotyping between Satt009 and Satt530, SSR were identified from the Williams 82 soybean genome sequence Glyma01 (Schmutz et al. 2010) and primers were designed. After PCR amplification, the SSRs that showed

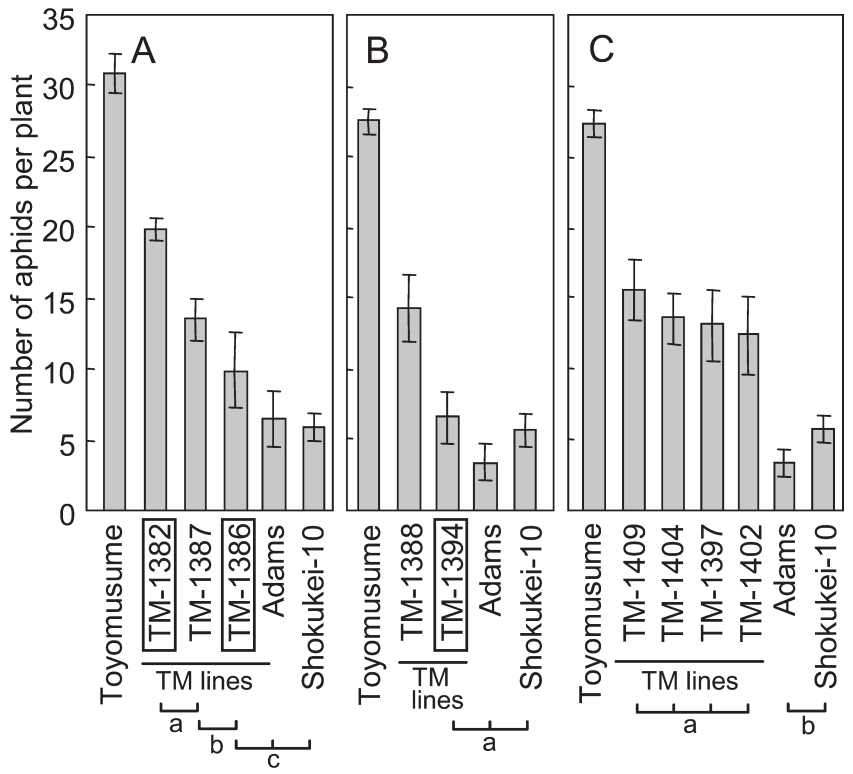

Fig. 2. Aphid resistance phenotype of Rasol-introduced Toyomusumebackcrossed lines (represented by TM lines). (A), (B) and (C) show the results of three independent experiments. Shokukei-10 is an Adams-derived breeding line that shows both foxglove aphid-resistant and SbDV-tolerant phenotypes. Vertical axis indicates the number of aphids per plant 14 days after settlement in a no-choice test. TM lines highlighted in a box were evaluated for their virus tolerance phenotype (see Figs. 2, 3). Vertical bars indicate the standard error of the means $(n=8)$. Static comparisons were carried out independently for each experiment. Grouping by the square brackets below the line names indicates there was no significant difference within the group at the 5\% level using the Tukey-Kramer multiple comparison test.

polymorphism between Adams and Shokukou-05405-1-1 or Toyoharuka, the parents of the mapping populations, were used as SSR markers. The position of the SSR markers on chromosome 3 and the sequences of the primers are shown in Table 1. The genotype of Satt641 and the newly developed SSR makers were assessed for 42 selected lines from 93 plants in which the recombination event was confirmed. For genotype data, 15 informative lines were used to evaluate the degree of aphid antibiosis. For screening of the mapping population, DNA extraction and SSR marker assay were performed as described previously (Sayama et al. 


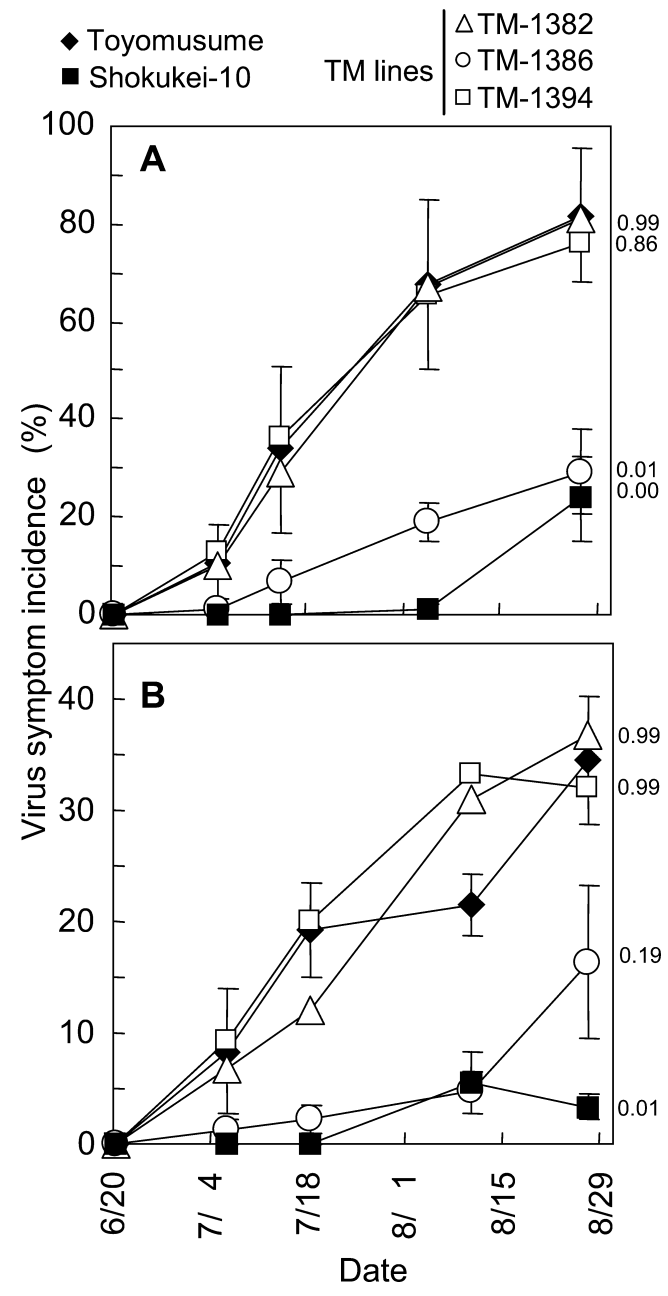

Fig. 3. SbDV tolerance phenotype of Toyomusume-backcrossed (TM) lines in the field. Virus symptom incidence in 2008 (A) and 2009 (B) are shown. Dwarfing or yellowing symptoms induced by SbDV were examined in the field. The $\mathrm{X}$-axis represents the assessment date. Vertical bars indicate the standard error of the means $(n=4)$. The standard errors for TM-1382 and TM-1394 are of the same level as Toyomusume and its display are omitted. The p-values by Dunnett's test are indicated on the right side of each panel. Dunnett's test was performed for the final observations in each year using Toyomusume as the reference.

2011). To obtain the sequence and gene annotation data of Williams 82, Phytozome v7.0 was used (Schmutz et al. 2010).

\section{Results}

\section{Aphid resistance of Raso1-introduced backcross lines}

Adams and its derivatives demonstrated antibiosis to foxglove. We used the term "aphid resistance" for this antibiosis. The foxglove aphid resistance QTL, Raso1, localized to a $3.7 \mathrm{cM}$ interval between Satt009 and Satt530 (Kamiya et al. 2008), was introduced into Toyomusume by backcrossing (Fig. 1). In all TM-lines tested, the number of aphids per plant was significantly lower than that observed on

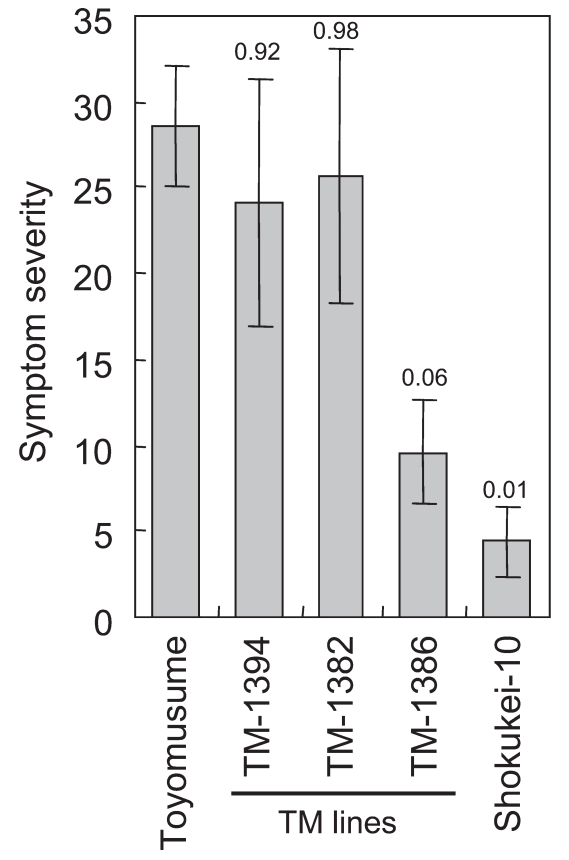

Fig. 4. Virus symptom severity of Toyomusume-backcrossed (TM) lines 118 days after sowing in 2009. Dwarfing or yellowing symptoms induced by SbDV in the field were assessed using the scoring system of Mikoshiba et al. (1990). Each plant was scored on a scale between 0 (no symptom) and 4 (severe symptoms) with increments of 1 . The symptom severity was calculated by [100(Sum of the score of plant)/ 4 (total number of plant)]. Vertical bars indicate the standard error of the means $(n=4)$. The p-values by Dunnett's test using Toyomusume as the reference are indicated at the top of the bars.

Toyomusume 14 days after aphid settlement (Fig. 2). However, the degree of aphid antibiosis varied among lines. TM-1386 and TM-1394 exhibited a similar number of aphids per plant as compared to Adams and Shokukei-10, from which the aphid resistance originated, whereas the other TM-lines exhibited a higher number of aphids than Adams did (Fig. 2). However, we concluded that the aphidresistance QTL Raso1 solely confers the aphid resistance phenotype when it is introduced by backcrossing.

\section{SbDV tolerance of Raso1-introduced backcross lines}

Adams and its derivatives demonstrated lower incidences of SbDV symptoms in the field. We used the term "SbDV tolerance" for this partial resistance in the field. The SbDV tolerance of 3 independent backcross lines differing in aphid resistance phenotype (TM-1382, TM-1386 and TM-1394, highlighted in boxes in Fig. 2) was tested. Only TM-1386 demonstrated virus tolerance equal to that of Shokukei-10, and TM-1382 and TM-1394 exhibited similar levels of virus symptom incidence as Toyomusume, the recurrent parent of both lines. Similar results were obtained in consecutive years (Fig. 3A, 3B) and when tolerance was evaluated as severity of virus symptoms (Fig. 4). 
A

Raso1

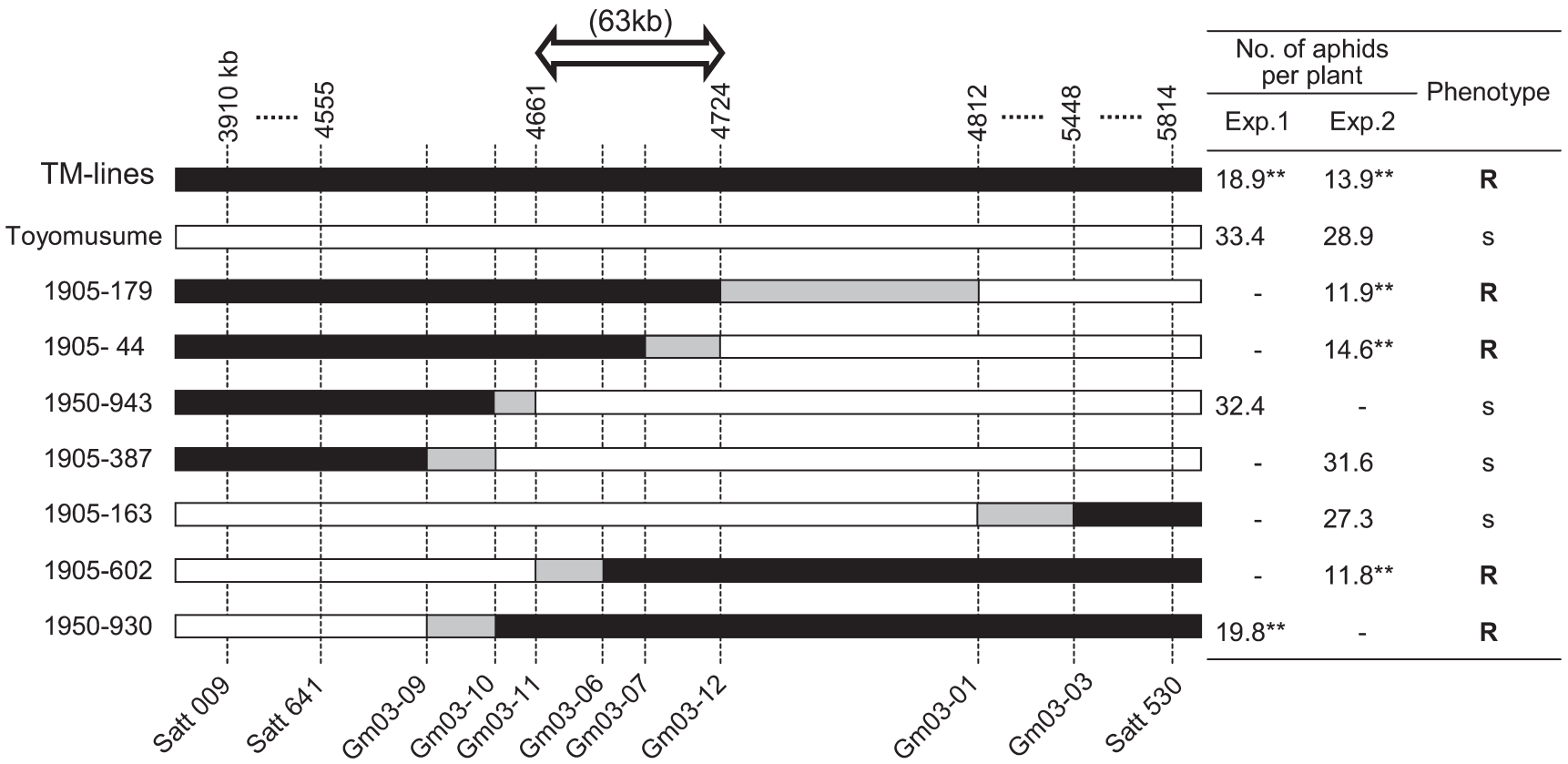

Fig. 5. Fine mapping of Rasol to a 63-kb interval of chromosome 3. (A) Summary of the informative seven lines used to fine-map the location of Raso1. Line names beginning with 1905 and 1950 indicate their derivation from the mapping populations C1905 and C1950, respectively. TMlines represent Toyomusume-backcrossed lines. Solid bars represent the genomic region derived from Adams, the source of aphid resistance. Open bars indicate the genomic region derived from Toyomusume or Toyoharuka or Shokukou-05405-1-1, the parent of the populations. Gray bars indicate intervals in which recombination events might occur. The scale at the top shows positions in the soybean genome sequence assembly (Glyma1) on chromosome 3. Intervals marked with horizontal dotted lines are greatly reduced in scale. Markers used for mapping (shown at the bottom) are described in Table 1. (B) Phenotype data of lines used for fine-mapping. The data represent the number of aphids per plant 14 days after settlement. For TM-lines, the results of bulked Toyomusume-backcrossed lines are shown. Jugged phenotype by aphid numbers are drawn at the rightmost column. Susceptible and resistant phenotype are abbreviate to ' $\mathrm{s}$ ' and ' $\mathrm{R}$ ' respectively. Significant differences from Toyomusume obtained with Dunnett's test at the $1 \%$ level are indicated by **.

\section{Fine mapping of Rasol}

Because Rasol solely showed the aphid resistance, finemapping of Rasol was undertaken. A total of $1245 \mathrm{~F}_{2}$ lines were screened for recombination events between the markers Satt009 and Satt530. Recombination events were confirmed in 93 lines, from which 42 lines were selected. The marker genotype for Satt641 and additional SSR markers located between Satt009 and Satt530 (Table 1) were determined for the 42 lines. For genotyping, Satt641 and additional SSR markers listed in Table 1 were used. Fifteen informative lines were selected and the degree of aphid antibiosis was investigated. The results for the seven mostinformative lines is presented in Fig. 5. Rasol was located between markers Gm03-11 and Gm03-12, comprising a $63 \mathrm{~kb}$ interval in the Williams 82 genome sequence. This interval contains three predicted genes in the Williams 82 genome sequence, namely Glyma03g04500 (TATA-box binding protein), Glyma03g04510 (calcium/calmodulin-dependent protein kinase-related) and Glyma03g04530 (NB-ARC domain and leucine-rich repeat-containing protein, a member of the nucleotide-binding site-leucine-rich repeat [NBSLRR] gene family) based on Phytozome v7.0.

\section{Discussion}

We introduced the aphid resistance QTL Rasol into a Toyomusume background by backcrossing. All Rasolintroduced lines showed the aphid resistance (Fig. 2). Hence we conclude Raso1 represents a resistance gene(s) effective against foxglove aphid. However, most backcross lines showed a lower degree of aphid antibiosis compared with the donor of Raso1, Shokukei-10, or Adams. While we observed less than 7 aphids per plant 14 days after settlement in Shokukei-10 and Adams, we observed more than 13 aphids in most TM-lines (Fig. 2). These observations imply the presence of additional minor gene(s) that modify the degree of aphid antibiosis in Adams.

In contrast to aphid resistance, introduction of Rasol by backcrossing was insufficient to exhibit the SbDV tolerance phenotype. Two backcross lines (TM-1382 and TM-1394) showed virus symptom incidence similar to that of the recurrent parent Toyomusume, even though they harbor Rasol (Fig. 3). Interestingly, only one line (TM-1386) exhibited a SbDV-tolerant phenotype (Fig. 3). Araki et al. (1997) reported that resistance to foxglove aphids was necessary for SbDV tolerance in segregated progenies of Adams. Our observations, together with those of Araki et al. (1997), suggest 
that an additional gene(s) is required for $\mathrm{SbDV}$ tolerance. Nevertheless, because the SbDV-tolerant line TM-1386 showed similar agricultural traits as the recurrent parent Toyomusume, it is useful for breeding programs in northern Japan.

Rasol was fine-mapped to a 63-kb fragment containing three gene candidates in the Williams 82 sequence assembly (Glyma1). One of the three gene candidates is a NB-ARC domain and leucine-rich repeat-containing gene (NBSLRR), a typical type of so-called R-gene. In this study only sequence data for the soybean Williams 82 was utilized. The corresponding genomic sequence in Adams is unknown, hence we cannot discuss further. However, this NBS-LRR gene is a most likely candidate of Rasol because the previously cloned aphid resistance genes Mi (Milligan et al. 1998, Rossi et al. 1998) and Vat (Dogimont et al. 2010), and candidates of the aphid resistance genes $A K R$ (Klingler et al. 2005), Rag1 (Kim et al. 2010a) and Rag2 (Kim et al. 2010b), are members of the NBS-LRR gene family.

In soybean, three resistance genes for soybean aphid (Aphis glycines) are reported, namely Ragl on chromosome 7 (Kim et al. 2010a), Rag2 (Kim et al. 2010b) and Rag3 (Zahng et al. 2010) on chromosome 13. We mapped Rasol on chromosome 3, therefore Rasol represents a gene independent from Rag1, Rag2 and Rag3. Moreover, previous reports demonstrate Adams does not show resistance to soybean aphid (Honda 1999), for which Rag1, Rag2 and Rag3 show resistance. Interestingly, candidates of Rag1 and Rag2 are NBS-LRR genes, the same type of gene as one of the candidates of Raso1. Thus soybean might evolve novel NBS-LRR genes for resistance to different aphid species. Comparisons among Raso1, Rag1 and Rag2 will help to improve our understanding of aphid resistance effected by the NBS-LRR gene family.

In the case of Adams, aphid resistance and tolerance to the virus transmitted by the aphid are closely related. A similar case was reported in melon with melon-cotton aphid (Aphis gossypii) and Cucumber mosaic virus (CMV) (Boissot et al. 2010, Pitrat et al. 1980, Sarria et al. 2008). In this case, the causal gene Vat, which shows both aphid resistance and the resistance to aphid transmission of CMV, was cloned and demonstrated to be a member of the NBS-LRR gene family (Dogimont et al. 2010). It is interesting to determine whether the aphid resistance manifested by NBS-LRR proteins will basically beside the resistance to virus transmission by the aphid or not.

\section{Acknowledgments}

This research was supported by the Ministry of Agriculture, Forestry and Fisheries of Japan [Genomics for Agricultural Innovation (DD-3211 and in part DD-3260), and in part by the Development of mitigation and adaptation techniques to global warming in the sectors of agriculture, forestry and fisheries (2002)].

\section{Literature Cited}

Araki,K., H.Jinno, K.Shirai, S.Hagihara and Y.Shinada (1997) Breeding highly tolerant lines to Soybean dwarf virus; relation between foxglove aphid resistance and soybean dwarf virus tolerance. Report of the Hokkaido Branch, the Japanese Society of Breeding and Hokkaido Branch, the Crop Science Society of Japan 38: 114-115.

Boissot, N., S. Thomas, N.Sauvion, C.Marchal, C.Pavis and C. Dogimont (2010) Mapping and validation of QTLs for resistance to aphids and whiteflies in melon. Theor. Appl. Genet. 121: 9-20.

Brotman, Y., L. Silberstein, I.Kovalski, C.Perin, C. Dogimont, M. Pitrat, J.Klingler, G. Thompson and R.Perl-Treves (2002) Resistance gene homologues in melon are linked to genetic loci conferring disease and pest resistance. Theor. Appl. Genet. 104: 10551063.

Dogimont,C., A.Bendahmane, V.Chovelon and N.Boissot (2010) Host plant resistance to aphids in cultivated crops: Genetic and molecular bases, and interactions with aphid populations. C. R. Biol. 333: 566-573.

Gildow,F.E., V.D.Damsteegt, A.L.Stone, O.P.Smith and S.M.Gray (2000) Virus-vector cell interactions regulating transmission specificity of soybean dwarf luteoviruses. J. Phytopathology 148: 333342 .

Honda, K. (1999) Characteristic of aphid resistance of Adams. Jpn. Soc. Appl. Ent. Zool. 43: 162.

Jinno,H., K.Araki, K.Kira and Y.Shinada (1997) Breeding highly tolerant lines to Soybean dwarf virus; with foxglove aphid resistance. Report of the Hokkaido Branch, the Japanese Society of Breeding and Hokkaido Branch, the Crop Science Society of Japan 38: 112113.

Kamiya, M., J. Sasaki, M. Saitou, O. Kanehira, Y.Hashimoto, T. Miyoshi, Y.Tanaka, F.Kousaka, S.Ohnishi, A. Tazawa, et al. (2008) QTL analysis and marker development of resistance in soybean to Aulacorthum solani, the aphid vector of Soybean dwarf virus (SbDV). Breed. Res. 10 (Suppl. 1): 146.

Kanehira, O. (1978) Seasonal Prevalence of Foxglove aphid Acyrthosiphon solani (KALTENBACH) in soybean varieties; differences in resistance to the soybean dwarf disease. Bull. Hokkaido Prefect. Agric. Exp. Stn. 40: 51-60.

Kim, K.S., S.Bellendir, K.A.Hudson, C.B.Hill, G.L.Hartman, D.L. Hyten, M.E.Hudson and B.W.Diers (2010a) Fine mapping the soybean aphid resistance gene Ragl in soybean. Theor. Appl. Genet. 120: 1063-1071.

Kim, K.S., C.B.Hill, G.L.Hartman, D.L.Hyten, M.E.Hudson and B.W.Diers (2010b) Fine mapping of the soybean aphid-resistance gene Rag2 in soybean PI 200538. Theor. Appl. Genet. 121: 599610.

Klingler,J., R.Creasy, L.Gao, R.M.Nair, A.S.Calix, H.S.Jacob, O.R. Edwards and K.B.Singh (2005) Aphid resistance in Medicago truncatula involves antixenosis and phloem-specific, inducible antibiosis, and maps to a single locus flanked by NBS-LRR resistance gene analogs. Plant Physiol. 137: 1445-1455.

Liu,X.M., C.M.Friebe and B.R.Gill (2005) Molecular mapping and allelic relationships of Russian wheat aphid-resistance genes. Crop Sci. 45: 2273-2280.

Mikoshiba,Y., K.Honda and I.Fujisawa (1990) Difference on damage caused by soybean dwarf disease among several soybean cultivars in Yamase area. Tohoku Agric. Res. 43: 147-148.

Mikoshiba,Y., K.Honda, S.Kanematsu and I.Fujisawa (1995) Distribution of Soybean Dwarf Virus Strainsin Japan. Annals of the Phytopathological Society of Japan 61: 276. 
Milligan, S.B., J.Bodeau, J.Yaghoobi, I.Kaloshian, P.Zabel and V.M. Williamson (1998) The root knot nematode resistance gene $M i$ from tomato is a member of the leucine zipper, nucleotide binding, leucine-rich repeat family of plant genes. Plant Cell 10: 13071320 .

Mittal,S., L.S.Dahleen and D.Mornhinweg (2008) Locations of quantitative trait loci conferring Russian wheat aphid resistance in barley germplasm STARS-9301B. Crop Sci. 48: 1452-1458.

Pitrat,M. and H.Lecoq (1980) Inheritance of resistance to cucumber mosaic virus transmission by Aphis gossypii in Cucumis melo. Phytopathology 70: 958-961.

Rossi,M., F.L.Goggin, S.B.Milligan, I.Kaloshian, D.E.Ullman and V.M.Williamson (1998) The nematode resistance gene $\mathrm{Mi}$ of tomato confers resistance against the potato aphid. Proc. Natl. Acad. Sci. USA 95: 9750-9754.

Sarria,E., F.J.Yuste-Lisbona, F.J.Palomares, A.I.López-Sesé, M.L. Gómez-Guillamón and M.Pitrat (2008) Inheritance of tolerance to Aphis gossypii in C. melo TGR-1551 and its relation with resistance to virus transmission. Cucurbitaceae 2008-IX EUCARPIA Meeting (poster session).

Sayama,T., T.Y.Hwang, K.Komatsu, Y.Takada, M.Takahashi, S. Kato, H.Sasama, A.Higashi, Y.Nakamoto, H.Funatsuki et al. (2011) Development and application of a whole-genome simple sequence repeat panel for high-throughput genotyping in soybean.
DNA Res. 18: 107-115.

Schmutz,J., S.B.Cannon, J.Schlueter, J.Ma, T.Mitros, W.Nelson, D.L. Hyten, Q. Song, J.J.Thelen, J.Cheng et al. (2010) Genome sequence of the palaeopolyploid soybean. Nature 463: 178-183

Smith,C.M. and E.V.Boyko (2007) The molecular bases of plant resistance and defense responses to aphid feeding: current status. Entomol. Exp. Appl. 122: 1-16.

So, Y.S., H.C.Ji and J.L.Brewbaker (2010) Resistance to corn leaf aphid (Rhopalosiphum maidis Fitch) in tropical corn (Zea mays L.). Euphytica 172: 373-381.

Tamada,T. (1970) Aphid transmission and host range of soybean dwarf virus. Ann. Phytopathol. Soc. Jpn. 36: 266-274.

Tamada,T. (1973) Strains of soybean dwarf virus. Ann. Phytopathol. Soc. Jpn. 39: 27-34.

Tamada, T. (1975) Studies on the soybean dwarf disease. Rep. Hokkaido Pref. Agric. Exp. Stn. 25: 1-144.

Tanimura,Y. and T.Tamada (1976) Breeding for resistance to soybean dwarf disease 1. Resistance of soybean varieties to soybean dwarf disease. Bull. Hokkaido Prefect. Agric. Exp. Stn. 35: 8-17.

Thekkeveetil,T., H.A.Hobbs, Y.Wang and D.Kridelbaugh (2007) First report of soybean dwarf virus in soybean in northern Illinois. Plant Dis. 91: 1686.

Zhang,G., C.Gu and D.Wang (2010) A novel locus for soybean aphid resistance. Theor. Appl. Genet. 120: 1183-1191. 